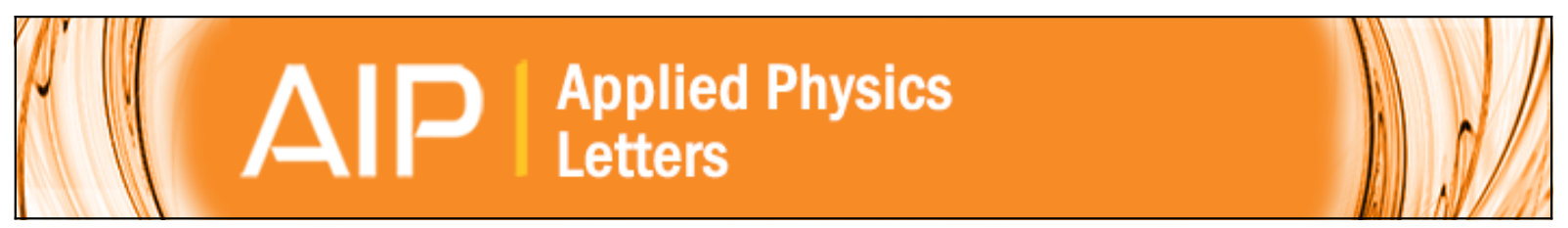

\title{
Nanosecond laser pulse heating of a platinum surface studied by pump-probe X-ray diffraction
}

Roman Shayduk, Vedran Vonk, Björn Arndt, Dirk Franz, Jörg Strempfer, Sonia Francoual, Thomas F. Keller,

Tobias Spitzbart, and Andreas Stierle

Citation: Applied Physics Letters 109, 043107 (2016); doi: 10.1063/1.4959252

View online: http://dx.doi.org/10.1063/1.4959252

View Table of Contents: http://scitation.aip.org/content/aip/journal/apl/109/4?ver=pdfcov

Published by the AIP Publishing

\section{Articles you may be interested in}

Erratum: "Reversible phase transition in laser-shocked 3Y-TZP ceramics observed via nanosecond time-resolved x-ray diffraction" [J. Appl. Phys. 111, 053526 (2012)]

J. Appl. Phys. 113, 039901 (2013); 10.1063/1.4776732

Picosecond acoustic response of a laser-heated gold-film studied with time-resolved $\mathrm{x}$-ray diffraction

Appl. Phys. Lett. 98, 191902 (2011); 10.1063/1.3584864

Nanosecond x-ray Laue diffraction apparatus suitable for laser shock compression experiments

Rev. Sci. Instrum. 81, 083902 (2010); 10.1063/1.3455211

Nanosecond x-Ray diffraction from polycrystalline and amorphous materials in a pinhole camera geometry suitable for laser shock compression experiments

Rev. Sci. Instrum. 78, 083908 (2007); 10.1063/1.2772210

In situ laser heating and radial synchrotron $\mathrm{x}$-ray diffraction in a diamond anvil cell

Rev. Sci. Instrum. 78, 063907 (2007); 10.1063/1.2749443

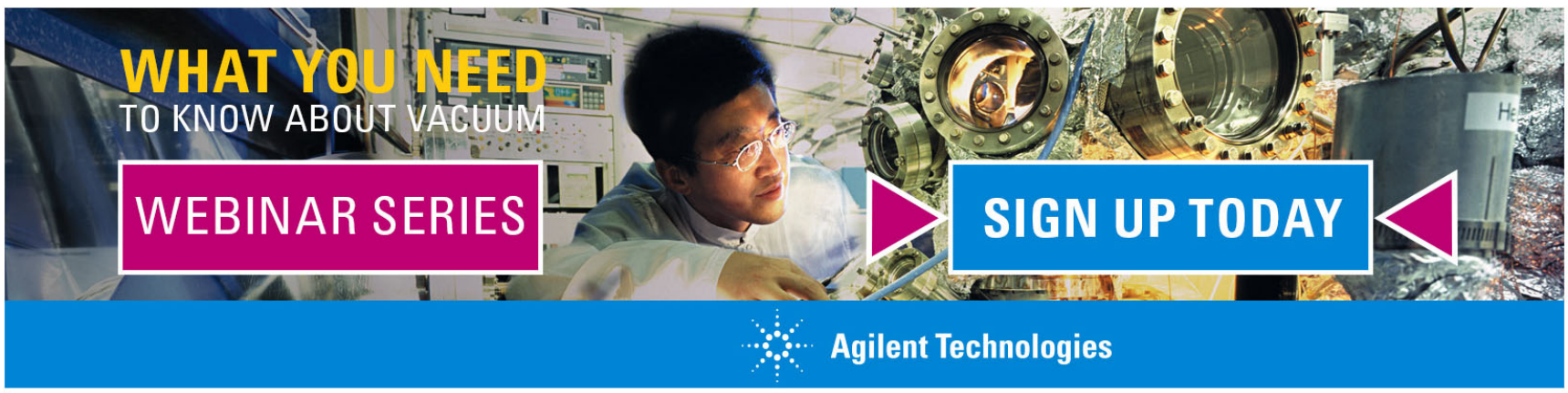




\title{
Nanosecond laser pulse heating of a platinum surface studied by pump-probe X-ray diffraction
}

\author{
Roman Shayduk, ${ }^{1}$ Vedran Vonk, ${ }^{1}$ Björn Arndt, ${ }^{1,2}$ Dirk Franz, ${ }^{1,2}$ Jörg Strempfer, ${ }^{1}$ \\ Sonia Francoual, ${ }^{1}$ Thomas F. Keller, ${ }^{1,2}$ Tobias Spitzbart, ${ }^{1}$ and Andreas Stierle ${ }^{1,2}$ \\ ${ }^{1}$ Deutsches Elektronen-Synchrotron DESY, D-22603 Hamburg, Germany \\ ${ }^{2}$ Fachbereich Physik, Universität Hamburg, D-20355 Hamburg, Germany
}

(Received 22 March 2016; accepted 9 July 2016; published online 29 July 2016)

\begin{abstract}
We report on the quantitative determination of the transient surface temperature of $\operatorname{Pt}(110)$ upon nanosecond laser pulse heating. We find excellent agreement between heat transport theory and the experimentally determined transient surface temperature as obtained from time-resolved X-ray diffraction on timescales from hundred nanoseconds to milliseconds. Exact knowledge of the surface temperature's temporal evolution after laser excitation is crucial for future pump-probe experiments at synchrotron storage rings and X-ray free electron lasers. Published by AIP Publishing. [http://dx.doi.org/10.1063/1.4959252]
\end{abstract}

As soon as a pulsed laser is used to excite a material, the challenge of transient surface temperature determination arises. Of interest is not only the initial temperature spike, but also the slower temperature decay, because this is related to the total pulse energy absorbed. This process is relevant for laser applications ranging from medicine and engineering to fundamental science. ${ }^{1-4}$ In industry nanosecond lasers are widely used in various micro-machining processes, such as material welding ${ }^{5}$ cutting, ${ }^{6}$ and ablation. ${ }^{7-11}$ The short-lived laser-induced temperature pulses in the heated region determine the conditions that may lead to material damage, phase transitions, and melting. In particular for pump-probe experiments, the goal is to only heat the sample and not to damage it. Therefore, it is indispensable to obtain reliable quantitative information about the surface temperature and possible laser-induced damage.

An important optical method for monitoring transient temperature due to laser excitation is transient thermoreflectance (TTR). ${ }^{12-17}$ With this method the effect of thermalinduced change of surface reflectance is employed to determine the surface temperature. ${ }^{12}$ For weak excitations the reflectance change is proportional to the temperature and the absolute temperature scale is determined by the so-called thermoreflectance coefficient, which is measured separately. ${ }^{18-20}$ For the detection of hot spots in microelectronic devices, TTR became a powerful tool which allows for a complete 2D-imaging of transient temperature fields of a microchip in operation. ${ }^{21-24}$

In this letter we present an alternative, complementary method to determine the transient laser-induced temperature by probing the effect of transient thermal lattice expansion. Since this method relies on X-ray diffraction, the underlying atomic-scale structure is directly probed and it delivers information from the surface and buried near-surface region. Such detailed high resolution information is complementary to the more macroscopic theory used to describe the optical surface in TTR. In addition, transient thermal lattice expansion plays a role in many laser-pump X-ray probe experiments at synchrotrons and X-ray Free Electron Lasers (XFELs), where structures are investigated down to picosecond timescales and in the future potentially with a spatial resolution of several tens of nanometers. ${ }^{25}$

We focus on a $\mathrm{Pt}(110)$ single crystal surface impulsively heated by a ns-laser pulse and compare the heating process on a quantitative basis with transient heat transport theory. Platinum is an important catalyst material used in exhaust cleaning, energy storage, and conversion technology. ${ }^{26}$ In this letter we demonstrate how to extract quantitative information on the temperature upon pulsed heating of Pt on nanosecond timescales using time-resolved $\mathrm{X}$-ray diffraction (TRXRD). Usually such experiments are done at large-scale facilities, such as synchrotron storage rings and X-ray Free Electron Lasers (XFELs). ${ }^{27-29}$ Here we show how to use a conventional continuous radiation X-ray tube to determine the laser-induced transient near-surface temperature.

In the experiment we used a $2 \mathrm{~mm}$ thick (110)-oriented polished Pt single crystal excited by a Nd:YAG actively Q-switched laser emitting at $1064 \mathrm{~nm}$. The laser delivers $7 \mathrm{~ns}$ pulses with energies up to $4 \mathrm{~mJ}$ at a maximum repetition rate of $1 \mathrm{kHz}$. A continuous $\mathrm{Cu}-\mathrm{K}_{\alpha} \mathrm{X}$-ray source, in combination with a $2 \mathrm{D}$ focussing multilayer optics delivering approximately $5 \times 10^{8}$ photons/s into a $0.25 \times 0.25 \mathrm{~mm}^{2}$ focus, was used.

The experiment was done in a stroboscopic pump-probe fashion at a repetition rate of $1 \mathrm{kHz}$, using a Pilatus $100 \mathrm{~K}$ (Ref. 30) X-ray area detector externally triggered. Each detector pixel worked in a gated single X-ray photon counting mode. The time resolution of the experiment was on the order of $100 \mathrm{~ns}$ due to the detector's sensor time response limitations. It is conceivable that other detector systems would allow for better time resolution. The detector gate signals were generated using a so-called Raspberry Pi Logic Controller (PiLC), which is a field-programmable gate arraybased unit developed at DESY. In the experiment the PiLC was synchronized with the laser emissions and was also used to delay the detector gate signal. Rough estimation of time zero, i.e., the point when the laser light and detector gate signal overlap, was determined using an oscilloscope via detection of the scattered laser light signal with an avalanche photo diode (APD) and the signal of the X-ray detector gate. 
Based on the work of Bechtel ${ }^{31}$ we first describe the semi-analytical solution to the heat equation relevant for our case.

We consider a laser pulse with Gaussian spatial and temporal profiles of irradiance at the solid surface:

$$
I(r, t)=\frac{E_{0}}{\tau} \frac{1}{\pi^{\frac{3}{2}} d^{2}} e^{-r^{2} / d^{2}-t^{2} / \tau_{0}^{2}},
$$

in which $E_{0}$ is the total pulse energy, $r$ is the radial coordinate of azimuthally homogeneous irradiance, and $\tau$ and $d$ are a measure of the pulse duration and laser beam size, respectively. Relation (1) defines time zero at the moment when half of the pulse has entered the solid and is normalized such that the total pulse energy integrated over space and time is given by $E_{0}$.

We define two different time regimes, which set boundary conditions on the heat equations. For time scales

$$
t \ll \tau_{1}=\frac{d^{2} C_{p} \rho}{4 k},
$$

the lateral heat diffusion can be neglected. Here $C_{p}, \rho$, and $k$ are the heat capacity, mass density, and heat conductivity coefficient of a solid, respectively. For our case (see Table I) $\tau_{1}$ evaluates to around $1 \mathrm{~ms}$. The finite volume of light absorption can be neglected for time scales longer than

$$
t \gg \tau_{2}=\frac{\alpha^{2} C_{p} \rho}{4 k},
$$

in which $\alpha$ is the light attenuation length. In the case of $\mathrm{Pt}$, using a value $\alpha=15 \mathrm{~nm}, \tau_{2}$ is of the order of ps. Our experimental conditions, using a ns-laser, are such that both conditions 2 and 3 are fulfilled. For this special case the temperature change field due to the pulsed laser excitation of a solid can be determined by evaluating the expression ${ }^{31}$

$$
\Delta T\left(r^{\prime}, z^{\prime}, t^{\prime}\right)=(1-R) \frac{E_{0}}{\pi^{2} d^{2}} \frac{1}{\sqrt{k C_{p} \rho \tau}} e^{-r^{\prime 2}} T^{\prime}\left(z^{\prime}, t^{\prime}, \lambda\right),
$$

in which $r^{\prime}, z^{\prime}$, and $t^{\prime}$ are dimensionless radial, depth, and time coordinates, respectively, and $\lambda$ is a material specific dimensionless parameter, all together defined as

$$
r^{\prime}=r / d ; \quad z^{\prime}=z / d ; \quad t^{\prime}=t / \tau ; \quad \lambda=\frac{2}{d} \sqrt{\frac{k \tau}{C_{p} \rho}} .
$$

\begin{tabular}{|c|c|c|c|}
\hline Physical property & Symbol & Value & Units \\
\hline Heat conductivity coefficient ${ }^{32}$ & $k$ & 73 & $\mathrm{~W} \mathrm{~m}{ }^{-1} \mathrm{~K}^{-1}$ \\
\hline Heat capacity ${ }^{33}$ & $C_{p}$ & 133 & $\mathrm{~J} \mathrm{~kg}^{-1} \mathrm{~K}^{-1}$ \\
\hline Mass density & $\rho$ & 22.42 & $\mathrm{~g} / \mathrm{cm}^{3}$ \\
\hline Reflectance at $1064 \mathrm{~nm}$ (Refs. 34 and 35) & $R$ & $0.78 \pm 0.03$ & $\ldots$ \\
\hline Pulse duration & $\tau$ & $7 \pm 0.2$ & ns \\
\hline Pulse energy & $E_{0}$ & $4 \pm 0.05$ & $\mathrm{~mJ}$ \\
\hline Focus diameter & $d$ & $1.2 \pm 0.1$ & $\mathrm{~mm}$ \\
\hline Pt thermal expansion ${ }^{36}$ & $\beta$ & $9 \times 10^{-6}$ & $\mathrm{~K}^{-1}$ \\
\hline Pt linear X-ray absorption ${ }^{37}$ & $\mu$ & $4.4 \times 10^{3}$ & $\mathrm{~cm}^{-1}$ \\
\hline
\end{tabular}

TABLE I. Physical properties of Pt and experimental conditions for which calculations of temperature profiles were performed.
The so-called dimensionless temperature change field $\Theta$ is a non-analytical function defined by the integral

$$
T^{\prime}\left(z^{\prime}, t^{\prime}, \lambda\right)=\int_{-\infty}^{t^{\prime}} \frac{\exp \left(-\xi^{2}-\frac{z^{\prime 2}}{\lambda^{2}} \frac{1}{t^{\prime}-\xi}\right)}{\sqrt{t^{\prime}-\xi}} \mathrm{d} \xi .
$$

Relation (4) is evaluated numerically using the parameters listed in Table I and the result is shown in Figure 1(b). The peak surface temperature is reached at $t \approx 0.55 \tau$ and is given by ${ }^{31}$

$$
T_{\max } \approx 0.22(1-R) \frac{E_{0}}{d^{2}} \frac{1}{\sqrt{k C_{p} \rho \tau}}
$$

which for our experimental conditions yields a peak surface temperature change of $108 \mathrm{~K}$ approximately at $t=4 \mathrm{~ns}$.

In case of isotropic quasi-static thermal expansion the local strain (local temperature-induced change in lattice spacing $a$ ) is directly proportional to the local temperature change via the thermal expansion coefficient $\beta$

$$
\epsilon(z, t)=\frac{\Delta a(z, t)}{a}=\beta \Delta T(z, t),
$$

where $\epsilon$ indicates the strain, which follows a particular nonuniform time-dependent temperature depth profile (z-profile), as indicated in Fig. 1. Essentially, the strain is maximum at the surface and then gradually vanishes into the bulk. The $\mathrm{X}$-ray beam is attenuated by the $\mathrm{Pt}$ and the contribution from different depths to the diffraction signal differs. The resulting Bragg peak position is determined by the absorptionweighted average and is a measure of the average strain $\bar{\epsilon}(t)$. Due to the imperfectness of the Pt crystal and the divergence of the X-ray beam the primary extinction can be neglected and therefore the incident X-ray beam is attenuated due to

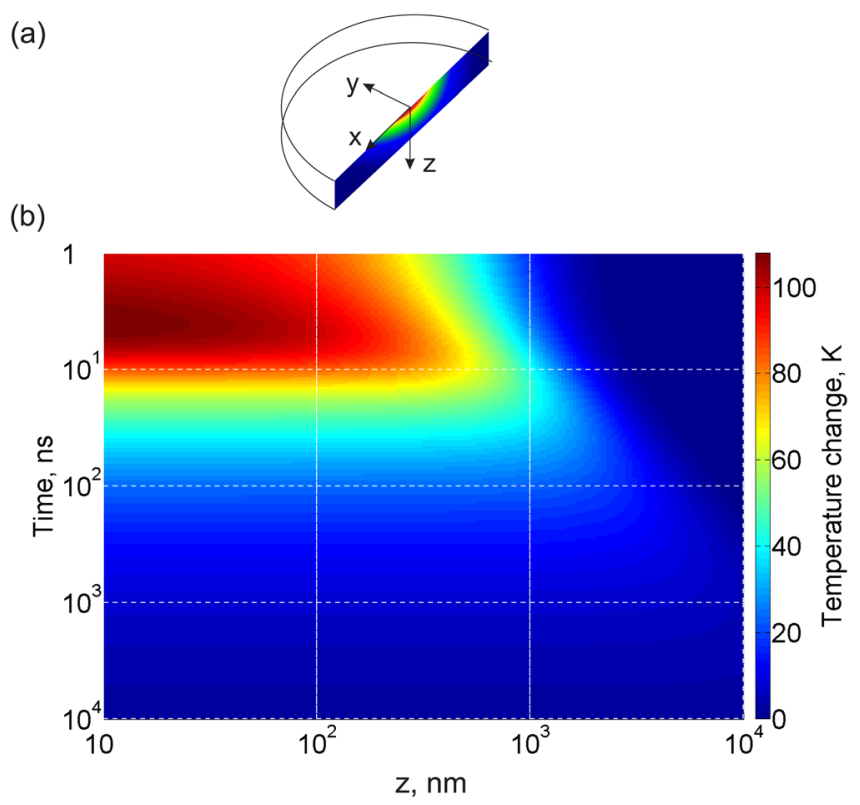

FIG. 1. (a) Sketch of the sample geometry with coordinate system placed in the center of the laser hot spot. (b) Temperature profile along the z-axis into the sample at different time moments after excitation calculated by the formula (4) for the experimental conditions and sample properties listed in Table I. 
the X-ray absorption. For the "extended face imperfect crystal" diffraction geometry used in the experiment, ${ }^{38} \bar{\epsilon}(t)$ is given by

$$
\bar{\epsilon}(t)=\langle\epsilon(z, t)\rangle_{z}=\frac{2 \mu}{\sin \left(\theta_{0}\right)} \int_{0}^{\infty} \epsilon(z, t) e^{-2 \mu z / \sin \left(\theta_{0}\right)} \mathrm{d} z
$$

with $\mu$ the linear absorption co-efficient, listed in Table I, and $\theta_{0}$ the Bragg angle of the symmetric (220) reflection.

Experimentally, from the time-dependent measured Bragg peak shift $\Delta \theta(t)$, the change in average strain $\bar{\epsilon}(t)$ is determined and thus the temperature. Whereas Eq. (9) describes the spatial averaging which occurs in the diffraction process, the finite experimental time resolution leads to an averaging in the time domain as well. The experimentally observed peak shift $\theta_{\text {exp }}$ is, through Bragg's law, related to the average strain convolved with a finite temporal resolution function $g(t)$ leading to

$$
\frac{\Delta \theta_{\exp }(t)}{\tan \left(\theta_{0}\right)}=-C \int_{-\infty}^{\infty} \bar{\epsilon}\left(t^{\prime}\right) g\left(t-t^{\prime}\right) \mathrm{d} t^{\prime} .
$$

For simplicity we use a unit-area Gaussian function for $g(t)$ with a fitting parameter $w$ describing the full width at half maximum (FWHM) of the Gaussian profile. This constant accounts for the detector gate width and for time jitters of all electronic components involved. A dimensionless scale constant $C$ is introduced to obtain the best agreement between the theoretically calculated temperature and the experimentally measured quantity. This constant accounts for uncertainties in the physical constants listed in Table I which determine the conditions of the experiment. Relation (10) is fitted to the experimental data using two parameters $C$ and $w$. In our experiment the temperature probing depth is equal to $\sin \left(\theta_{0}\right) / 2 \mu$ which is around $500 \mathrm{~nm}$.

Figure 2(a) shows two Pt (220) Bragg peak profiles measured at different times with respect to the laser pulse arrival. By varying the detector gate delay with respect to the laser emissions we recorded the Bragg peak profiles at different delays after the excitation pulse. Fig. 2(b) indicates the resulting relative peak positions and peak widths changes obtained from fits using a Pseudo-Voigt function. The maximum Bragg peak width, shown in Fig. 2(b), was detected at different delays compared with the maximum observed peak shift. The measured Bragg peak's width stems from the $\mathrm{X}$-ray beam divergence function convoluted with the intrinsic Bragg profile. The intrinsic Bragg peak profile depends on multiple factors as crystal mosaicity, static strain due to dislocations, and dynamic strain due to the temperature gradients. ${ }^{39,40}$ The X-ray beam divergence of the set-up is much larger than any of the other effects mentioned to give rise to intrinsic Bragg peak broadening. That is, in the region $-100 \mathrm{~ns}<\Delta t<100 \mathrm{~ns}$, the measured Bragg peak profile consists of both the pumped and un-pumped states. Since the two associated signals are shifted in position, the total measured profile appears to be broadened. The maximum width corresponds to the gate delay when the 7 ns-long laser pulse

(a)

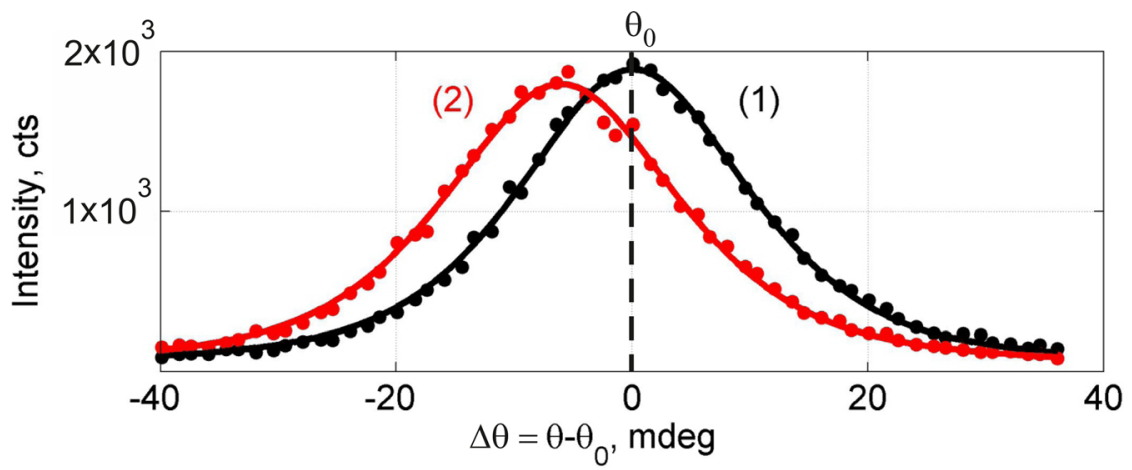

(b)

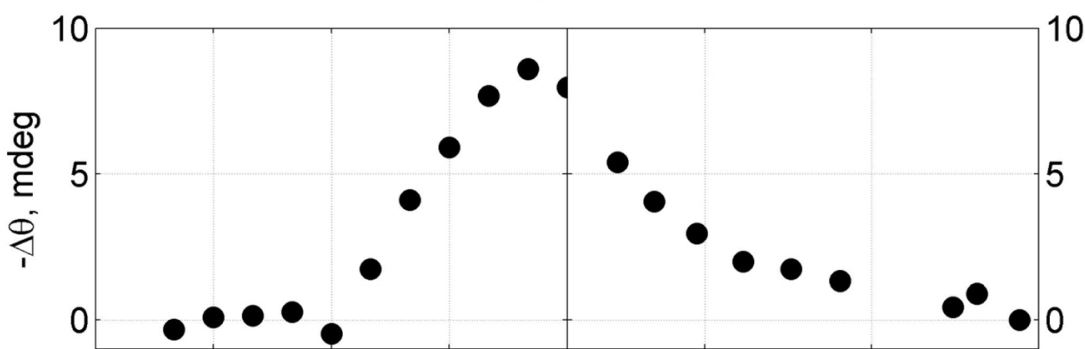

FIG. 2. (a) Plot of (220)-Bragg peak profiles with corresponding fitted Pseudo-Voigts. The black curve corresponds to the negative gate delay of $-150 \mathrm{~ns}$ and the red curve corresponds to the positive gate delay of $150 \mathrm{~ns}$. (b) Plot of the Bragg peak shift as a function of gate delay. (c) Plot of the peak FWHM change as a function of gate delay.

(c)

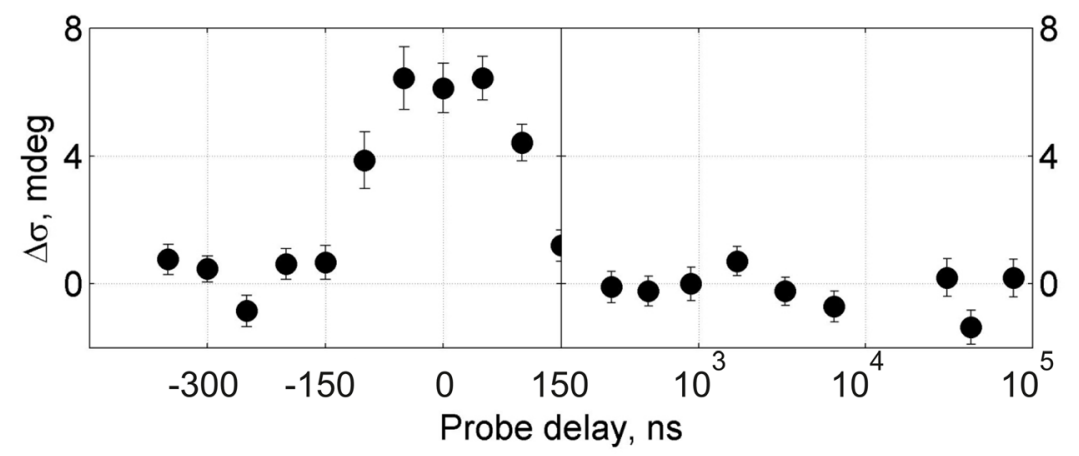


hits the center of the $100 \mathrm{~ns} \mathrm{X}$-ray detector gate. This effect allows to determine time zero with a significantly better accuracy than the $100 \mathrm{~ns}$ gate width.

The best fit of (4) to the observed transient Bragg peak positions is obtained for the following fitting parameters: $w=89 \mathrm{~ns}$ and $C=1.06$. The parameter $w$ describing the time resolution of the experiment is close to the Pilatus $100 \mathrm{~K}$ single pixel response time.

The blue curve in the Fig. 3 is the plot of the fit function (10) and the red solid line is the calculated surface temperature (4) multiplied by the factor of $C$. The right axis of the plot in Fig. 3 is obtained by the use of Eq. (8) and shows the lattice temperature change with time. At timescales longer than 300 ns after the excitation the blue and the red curves coincide, meaning that temperature is homogeneous within the probing depth (see Fig. 1(b)) and the cooling rate is slow enough to be resolved in this experiment. Therefore, in this time regime, no time convolution or space averaging is necessary to reproduce the data. The temperature tail in this experiment provides the most direct quantitative information on the near-surface temperature of the crystal due to the pulsed laser heating on nanosecond timescales. The laser induced temperature on nanosecond timescale is also relevant for ps and fs pulse excitations because it contains quantitative information about the total absorbed energy of the pulse.

The obtained results demonstrate that the quasi-static description of pulsed-laser-induced heating gives an adequate description of the experimental results in the ns to ms time-regime. This means that one can safely neglect the formation of thermoelastic waves, which manifest themselves on different time-scales. ${ }^{40}$

In the proposed method the near-surface temperature is determined indirectly by measuring the strain which can be detected ${ }^{27}$ with the sensitivity better than $10^{-7}$ corresponding to the uncertainty of the temperature change detection of $0.01 \mathrm{~K}$. However, the accuracy of the temperature determination essentially depends on the accuracy of the linear expansion coefficient $\beta$ used for the strain-temperature mapping. In

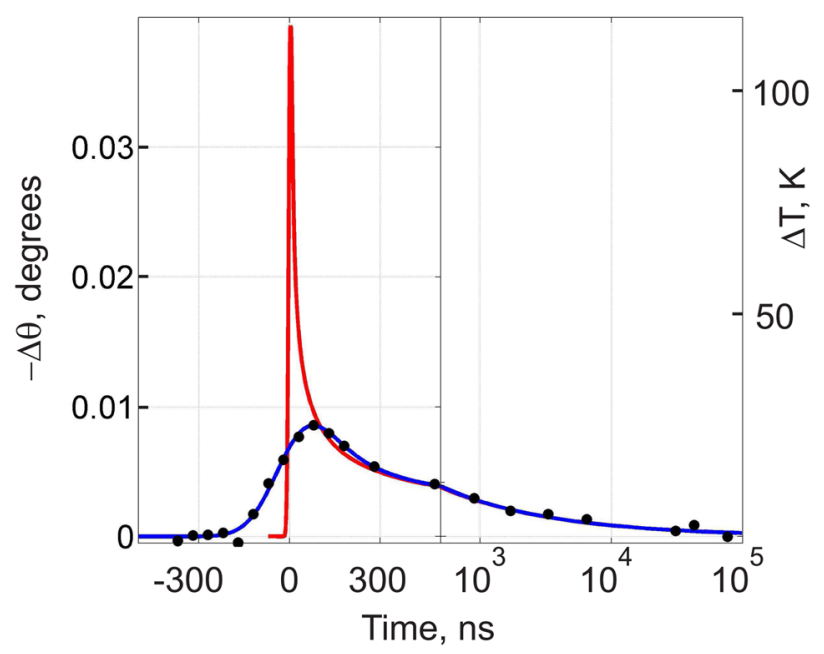

FIG. 3. Blue solid line is the plot (with abscissa on the left) of (10) and red solid line is the plot (with abscissa on the right) of (4) multiplied by a factor of $C$. The black dots are experimental points. On the right hand side the scale is shown in Kelvin obtained from (8). The estimated experimental errors in Kelvin correspond to around $2 \mathrm{~K}$. this letter we assumed an isotropic approximation of the thermal expansion. In case of an anisotropic thermal expansion, ${ }^{41}$ the single linear thermal expansion coefficient may not be used. The anisotropy of thermal expansion can be investigated in future experiments employing an asymmetric diffraction geometry. To improve the surface sensitivity the experiment could be done in grazing incidence or exit geometry.

The fact that the experimental data are well reproduced on all measured timescales with the single time-independent scale constant $C$ points to an adequate description of the measured strain dynamics in the framework of quasi-static thermal expansion on sub-microsecond timescales.

In summary, we determined the transient near-surface temperature of $\mathrm{Pt}(110)$ heated by ns-laser pulses from TRXRD in combination with an isotropic quasi-static thermoelastic theory. The temperature is followed on timescales from $100 \mathrm{~ns}$ to $0.1 \mathrm{~ms}$ after the laser pulse impinges on the sample. These experiments deliver very important quantitative information about the pulsed-laser-induced transient surface temperature and its decay. Knowledge about the exact temperature profile induced by a pulsed laser is essential in particular for systematic pump-probe experiments performed at synchrotron storage rings and XFELs. X-ray-diffraction-based techniques are complementary to all-optical methods and will further strengthen the arsenal of time-resolved thermographic tools.

We acknowledge Maria Teresa Nunez Pardo de Vera, Marcus Fleck, Andre Rothkirch, and Thorsten Kracht for assistance in setting up TANGO device server and software interface for controlling the synchronization electronics unit PiLC.

${ }^{1}$ B. Girard, D. Yu, M. R. Armstrong, B. C. Wilson, C. M. L. Clokie, and R. J. D. Miller, Lasers Surg. Med. 39, 273 (2007).

${ }^{2}$ M. Hess, M. D. Hildebrandt, F. Mueller, S. Kruber, P. Kroetz, U. Schumacher, R. Reimer, M. Kammal, K. Pueschel, W. Woellmer et al., Eur. Arch. Oto-Rhino-laryngol. 270, 2927 (2013).

${ }^{3}$ N. Jowett, W. Woellmer, A. M. Mlynarek, P. Wiseman, B. Segal, K. Franjic, P. Kroetz, A. Boettcher, R. Knecht, and R. J. D. Miller, JAMA Otolaryngol. Head Neck Surg. 139, 828 (2013).

${ }^{4}$ M. Dell'Angela, T. Anniyev, M. Beye, R. Coffee, A. Foehlisch, J. Gladh, T. Katayama, S. Kaya, O. Krupin, J. LaRue et al., Science 339, 1302 (2013).

${ }^{5}$ C. Luo and L. W. Lin, Sens. Actuators A-Phys. 97-98, 398-404 (2002).

${ }^{6}$ M. R. H. Knowles, G. Rutterford, D. Karnakis, and A. Ferguson, Int. J. Adv. Manuf. Technol. 33, 95 (2007).

${ }^{7}$ B. N. Chichkov, C. Momma, S. Nolte, F. vonAlvensleben, and A. Tunnermann, Appl. Phys. A 63, 109 (1996).

${ }^{8}$ C. Momma, B. N. Chichkov, S. Nolte, F. vonAlvensleben, A. Tunnermann, H. Welling, and B. Wellegehausen, Opt. Commun. 129, 134 (1996).

${ }^{9}$ T. V. Kononenko, S. V. Garnov, S. M. Klimentov, V. I. Konov, E. N. Loubnin, F. Dausinger, A. Raiber, and C. Taut, Appl. Surf. Sci. 109-110, 48-51 (1997).

${ }^{10}$ T. V. Kononenko, V. G. Ralchenko, I. I. Vlasov, S. V. Garnov, and V. I. Konov, Diamond Relat. Mater. 7, 1623 (1998).

${ }^{11}$ X. L. Chen and X. B. Liu, J. Laser Appl. 11, 268 (1999).

${ }^{12}$ M. Farzaneh, K. Maize, D. Luerssen, J. A. Summers, P. M. Mayer, P. E. Raad, K. P. Pipe, A. Shakouri, R. J. Ram, and J. A. Hudgings, J. Phys. D: Appl. Phys. 42, 143001 (2009).

${ }^{13}$ D. G. Cahill, P. V. Braun, G. Chen, D. R. Clarke, S. Fan, K. E. Goodson, P. Keblinski, W. P. King, G. D. Mahan, A. Majumdar et al., Appl. Phys. Rev. 1, 011305 (2014).

${ }^{14}$ R. Garrelts, A. Marconnet, and X. Xu, Nanoscale Microscale Thermophys. Eng. 19, 245 (2015). 
${ }^{15}$ V. M. Sundaram, A. Soni, and S.-B. Wen, J. Appl. Phys. 115, 234903 (2014).

${ }^{16}$ J. P. Feser and D. G. Cahill, Rev. Sci. Instrum. 84, 049901 (2013).

${ }^{17}$ F. Nakamura, N. Taketoshi, T. Yagi, and T. Baba, Meas. Sci. Technol. 22, 024013 (2011).

${ }^{18}$ S. Dilhaire, S. Grauby, and W. Claeys, Appl. Phys. Lett. 84, 822 (2004).

${ }^{19}$ M. G. Burzo, P. L. Komarov, and P. E. Raad, IEEE Trans. Compon. Packag. Technol. 28, 39-44 (2005).

${ }^{20}$ J. Christofferson, K. Maize, Y. Ezzahri, J. Shabani, X. Wang, and A. Shakouri, J. Electron. Packag. 130, 041101 (2008).

${ }^{21}$ A. Soni, V. M. Sundaram, and S.-B. Wen, Appl. Phys. Lett. 102, 203112 (2013).

${ }^{22}$ K. Maize, A. Ziabari, W. D. French, P. Lindorfer, B. OConnell, and A. Shakouri, IEEE Trans. Electron Devices 61, 3047 (2014).

${ }^{23}$ B. Vermeersch, J.-H. Bahk, J. Christofferson, and A. Shakouri, J. Appl. Phys. 113, 104502 (2013).

${ }^{24}$ B. Vermeersch, G. Pernot, H. Lu, J.-H. Bahk, A. Gossard, and A. Shakouri, Phys. Rev. B 88, 214302 (2013).

${ }^{25}$ C. Krywka, H. Neubauer, M. Priebe, T. Salditt, J. Keckes, A. Buffet, S. V. Roth, R. Doehrmann, and M. Mueller, J. Appl. Crystallogr. 45, 85 (2012).

${ }^{26}$ G. Ertl, Angew. Chem. Int. Ed. 47, 3524 (2008).

${ }^{27}$ R. Shayduk, H. Navirian, W. Leitenberger, J. Goldshteyn, I. Vrejoiu, M. Weinelt, P. Gaal, M. Herzog, C. V. K. Schmising, and M. Bargheer, New J. Phys. 13, 093032 (2011).
${ }^{28}$ H. Navirian, R. Shayduk, W. Leitenberger, J. Goldshteyn, P. Gaal, and M. Bargheer, Rev. Sci. Instrum. 83, 063303 (2012).

${ }^{29}$ H. A. Navirian, D. Schick, P. Gaal, W. Leitenberger, R. Shayduk, and M. Bargheer, Appl. Phys. Lett. 104, 021906 (2014).

${ }^{30}$ M. Bech, O. Bunk, C. David, P. Kraft, C. Bronnimann, E. F. Eikenberry, and F. Pfeiffer, Appl. Radiat. Isotopes 66, 474 (2008).

${ }^{31}$ J. H. Bechtel, J. Appl. Phys. 46, 1585 (1975).

${ }^{32}$ R. W. Powell and R. P. Tye, Br. J. Appl. Phys. 14, 662 (1963).

${ }^{33}$ H. Yokokawa and Y. Takahashi, J. Chem. Thermodyn. 11, 411 (1979).

${ }^{34}$ A. Y.-C. Yu and W. E. Spicer, Phys. Rev. 171, 834 (1968).

${ }^{35}$ T. Shiraishi, K. Hisatsune, Y. Tanaka, E. Miura, and Y. Takuma, Gold Bull. 34, 129 (2001).

${ }^{36}$ R. K. Kirby, Int. J. Thermophys. 12, 679 (1991).

${ }^{37} \mathrm{~J}$. Hubbell and S. Seltzer, Tables of X-ray mass attenuation coefficients and mass energy-absorption coefficients $1 \mathrm{keV}$ to $20 \mathrm{meV}$ for elements $\mathrm{z}=1$ to 92 and 48 additional substances of dosimetric interest, 1995.

${ }^{38}$ B. E. Warren, X-ray Diffraction (Dover, 1990).

${ }^{39}$ D. Schick, M. Herzog, H. Wen, P. Chen, C. Adamo, P. Gaal, D. G. Schlom, P. G. Evans, Y. Li, and M. Bargheer, Phys. Rev. Lett. 112, 097602 (2014).

${ }^{40}$ D. Schick, M. Herzog, A. Bojahr, W. Leitenberger, A. Hertwig, R. Shayduk, and M. Bargheer, Struct. Dyn. 1, 064501 (2014).

${ }^{41}$ M. Highland, B. C. Gundrum, Y. K. Koh, R. S. Averback, D. G. Cahill, V. C. Elarde, J. J. Coleman, D. A. Walko, and E. C. Landahl, Phys. Rev. B 76, 075337 (2007). 